\title{
NUMERICAL SOLUTION OF THE INCOMPRESSIBLE NAVIER-STOKES EQUATIONS WITH AN UPWIND COMPACT DIFFERENCE SCHEME
}

\author{
MA YANWEN ${ }^{\mathrm{a}, *}$, FU DEXUN $^{\mathrm{a}}$, T. KOBAYASHI ${ }^{\mathrm{b}}$ AND N. TANIGUCHI ${ }^{\mathrm{b}}$ \\ ${ }^{a}$ LNM, Institute of Mechanics, Chinese Academy of Sciences, 15 Zhong Guan cun Road, Beijing 100080, China \\ ${ }^{\mathrm{b}}$ Institute of Industrial Science, University of Tokyo, Tokyo, Japan
}

\begin{abstract}
SUMMARY
A new finite difference method for the discretization of the incompressible Navier-Stokes equations is presented. The scheme is constructed on a staggered-mesh grid system. The convection terms are discretized with a fifth-order-accurate upwind compact difference approximation, the viscous terms are discretized with a sixth-order symmetrical compact difference approximation, the continuity equation and the pressure gradient in the momentum equations are discretized with a fourth-order difference approximation on a cell-centered mesh. Time advancement uses a three-stage Runge-Kutta method. The Poisson equation for computing the pressure is solved with preconditioning. Accuracy analysis shows that the new method has high resolving efficiency. Validation of the method by computation of Taylor's vortex array is presented. Copyright (C) 1999 John Wiley \& Sons, Ltd.
\end{abstract}

KEY WORDS: finite difference scheme; compact difference method; higher-order scheme; Navier-Stokes equations; incompressible flow

\section{INTRODUCTION}

Recently, people have been paying more attention to numerical simulation of complex flow fields with multi-scale structures. A turbulent fluid flow is a common example. There is a need to perform direct numerical simulation instead of solving the averaged Navier-Stokes equations. Computational algorithms developed in the past were mainly designed for solving large scale fluid dynamics problems. For simulating the complex flow fields with multi-scale structures, a high resolution method is needed. There are two ways to improve the resolution of the method. One of them is to construct a high-order-accurate scheme, and another is to refine the grid mesh. With lower accuracy schemes (e.g. second-order schemes), it is difficult to capture small scale structures in a complex flow field like turbulence because of limitation of computer resources. Hence high-order-accurate computational methods are both desirable and preferred. In early studies spectral methods were used to directly simulate the turbulent flows $[1-3]$. Because of their flexibility and common use, finite difference methods have also recently

\footnotetext{
* Correspondence to: LNM, Institute of Mechanics, Chinese Academy of Sciences, 15 Zhong Guan cun Road, Beijing
} 100080, China.

Contract/grant sponsor: State Key Project; Contract/grant numbers: NNSFC and JSPS

Contract/grant sponsor: Network Information Centre; Contract/grant number: CAS

CCC 0271-2091/99/130509-13\$17.50

Received October 1997

Copyright (C) 1999 John Wiley \& Sons, Ltd.

Revised June 1998 
been used to solve the complex flow fields with multi-scale structures. Rai and Moin [4] present simulation of an incompressible turbulent channel flow using a fifth-order-accurate upwind-biased difference scheme. In Reference [5], symmetrical compact difference schemes are developed, and they are used to solve compressible mixing layers. In References [6,7], upwind compact difference schemes and a super compact finite difference method (SCFDM) are developed. With high-order-accurate schemes, the range of wavenumbers with accurate simulation can be enlarged. The smaller structures of fluid flow can be captured well with high-order schemes.

One of the main problems of turbulence simulation is to ensure that the effects of turbulent diffusion are not masked by numerical diffusion. For dominance of physical diffusion, the mesh Reynolds number, $\operatorname{Re} \Delta x$, must be restricted. In Reference [6], it was shown that for high-order-accurate schemes, the restriction on $\operatorname{Re} \Delta x$ can be relaxed.

Another important problem of turbulent simulation is to ensure that the phase speed of flow structures with different scales can be well-approximated. Otherwise the computed coherent structures will not be physical [8]. The commonly used second-order central schemes with a limited number of grid points cannot give correct phase speed for the structures with small scales. With higher-order-accurate schemes, the situation can be much improved.

Within the high-order-accurate schemes with the same order of accuracy, the compact and upwind compact schemes have higher resolving efficiency than the traditional schemes $[5,6]$, and the more complicated SCFDM has higher resolving efficiency than the compact schemes. In the present paper, the upwind compact scheme developed in Reference [6] is used to discretize the incompressible Navier-Stokes equations. The system of equations is discretized on a staggered mesh grid system. The fifth-order upwind compact difference approximation is used to discretize the convection terms in the momentum equations, and the viscous terms are discretized with a sixth-order symmetrical compact difference approximation. The derivatives in the continuity equation and the pressure gradient in the momentum equations are discretized with fourth-order accuracy on a cell-centered mesh. For advancing time a three-stage Runge-Kutta method with third-order accuracy is used [9]. The pressure is solved from the discretized Poisson equation, which is obtained by putting the discretized momentum equations into the discretized continuity equation. For solving the obtained pressure equation, preconditioning is used [10].

With the new developed scheme, the smaller structures in the complex flow fields can be well-captured. Compared with the fifth-order upwind-biased schemes in [4], the new method has less numerical dissipation in the wide range of wavenumbers, and larger numerical dissipation in the range with very high wavenumbers. The numerical dissipation with very high wavenumbers is useful for suppressing non-physical oscillations. In this paper, the discretization method for solving the incompressible Navier-Stokes equations is presented first, and then the accuracy of the scheme is analyzed. In Section 6, examples are given.

\section{GOVERNING EQUATIONS}

For two-dimensional flows, the governing equations describing incompressible flow in Cartesian co-ordinates in dimensionless form are

\section{Continuity equation}

$$
\frac{\partial u}{\partial x}+\frac{\partial v}{\partial y}=0 .
$$


Momentum equation in the horizontal direction

$$
\frac{\partial u}{\partial t}+u \frac{\partial u}{\partial x}+v \frac{\partial u}{\partial y}+\frac{\partial p}{\partial x}=\frac{1}{R e}\left(\frac{\partial^{2} u}{\partial x^{2}}+\frac{\partial^{2} u}{\partial y^{2}}\right)
$$

Momentum equation in the vertical direction

$$
\frac{\partial v}{\partial t}+u \frac{\partial v}{\partial x}+v \frac{\partial v}{\partial y}+\frac{\partial p}{\partial y}=\frac{1}{R e}\left(\frac{\partial^{2} v}{\partial x^{2}}+\frac{\partial^{2} v}{\partial y^{2}}\right)
$$

In these equations, the characteristic length is $L$, the characteristic velocity is $U_{0}$, and the characteristic time is $L / U_{0}$. The dimensionless pressure is $p=\bar{p} /\left(\rho U_{0}^{2}\right)$ in which $\rho$ is the density (constant), and $\bar{p}$ is the dimensional pressure. In the momentum equations, the parameter $R e=U_{0} L / \gamma$ is the Reynolds number in which $\gamma$ is the kinematic viscosity of the fluid.

The difficulty of solving the system of (2.1)-(2.3) is that the flow parameters are not in evolutionary form. The velocity components $u$ and $v$ in the numerical solution of the momentum equations at each time step must satisfy the discretized continuity equation. For the easy design of the solution algorithm, defining

$$
\begin{aligned}
& D(U)=\frac{\partial u}{\partial x}+\frac{\partial v}{\partial y}, \\
& L(U)=\left[f-\frac{\partial p}{\partial x}, g-\frac{\partial p}{\partial y}\right]^{T}, \\
& f=\frac{1}{R e}\left(\frac{\partial^{2} u}{\partial x^{2}}+\frac{\partial^{2} u}{\partial y^{2}}\right)-u \frac{\partial u}{\partial x}-v \frac{\partial u}{\partial y}, \\
& g=\frac{1}{R e}\left(\frac{\partial^{2} v}{\partial x^{2}}+\frac{\partial^{2} v}{\partial y^{2}}\right)-u \frac{\partial v}{\partial x}-v \frac{\partial v}{\partial y}, \\
& F=[f, g]^{T}, \quad U=[u, v]^{T}
\end{aligned}
$$

the following system of equations is obtained

$$
\begin{aligned}
& D(U)=0, \\
& \frac{\partial U}{\partial t}=L(U) .
\end{aligned}
$$

\section{DIFFERENCE APPROXIMATION}

The staggered grid point system is given in Figure 1. The first momentum equation is written at the point $\left(i+\frac{1}{2}, j\right)$, the second momentum equation is written at the point $\left(i, j+\frac{1}{2}\right)$, and the pressure is given at point $(i, j)$. The continuity equation is approximated at the point $(i, j)$. The convection terms in the momentum equations are discretized with a fifth-order-accurate upwind compact difference approximation [6]. For example, the term $u(\partial u / \partial x)$ is split as follows

$$
u \frac{\partial u}{\partial x}=u^{+} \frac{\partial u}{\partial x}+u^{-} \frac{\partial u}{\partial x}, \quad u^{+}+u^{-}=u, \quad u^{+} \geq 0, \quad u^{-} \leq 0 .
$$

The term $u^{+}(\partial u / \partial x)$ is approximated at point $\left(i+\frac{1}{2}, j\right)$ by $u_{i+1 / 2}^{+} F_{i+1 / 2}^{+} / \Delta x$ and

$$
\frac{3}{5} F_{i+1 / 2}^{+}+\frac{2}{5} F_{i-1 / 2}^{+}=\frac{1}{60} \delta_{x}^{-}\left[-u_{i+5 / 2}+11 u_{i+3 / 2}+47 u_{i+1 / 2}+3 u_{i-1 / 2}\right]
$$


the term $u^{-}(\partial u / \partial x)$ at point $\left(i+\frac{1}{2}, j\right)$ is approximated with $u_{i+1 / 2}^{-} F_{i+1 / 2}^{-} / \Delta x$ and

$$
\frac{3}{5} F_{i+1 / 2}^{-}+\frac{2}{5} F_{i+3 / 2}^{-}=\frac{1}{60} \delta_{x}^{+}\left[3 u_{i+3 / 2}+47 u_{i+1 / 2}+11 u_{i-1 / 2}-u_{i-3 / 2}\right],
$$

where $\delta_{\frac{x}{x}}^{ \pm} u_{i}=\mp\left(u_{i}-u_{i \pm 1}\right)$. Here $u^{ \pm}$and $F^{ \pm}$are defined at points $\left(i+\frac{1}{2}, j\right)$. Equations (3.1) and (3.2) can be solved easily. In the same way, difference approximations can be constructed for other convection terms in the momentum equations. The viscous terms are approximated with a sixth-order symmetric compact difference relation [5]. For example, the term $\partial^{2} u / \partial x^{2}$ is approximated with $S_{i+1 / 2} / \Delta x^{2}$ and

$$
\frac{2}{15} S_{i+3 / 2}+\frac{11}{15} S_{i+1 / 2}+\frac{2}{15} S_{i-1 / 2}=\frac{4}{5} \delta_{x}^{2} u_{i+1 / 2}+\frac{1}{20}\left[u_{i+5 / 2}-2 u_{i+1 / 2}+u_{i-3 / 2}\right],
$$

where the difference operator $\delta_{x}^{2}=\delta_{x}^{+} \delta_{x}^{-}$. To obtain $S_{i+1 / 2}$, a linear system of equations with a tri-diagonal matrix has to be solved.

The derivatives of pressure in the momentum equations are discretized with a fourth-order difference approximation on a cell-centered mesh. For example, at the point $\left(i+\frac{1}{2}, j\right)$, one has

$$
\delta_{x} p_{i+1 / 2}=\Delta x \frac{\partial p}{\partial x}=\frac{1}{24}\left[27\left(p_{i+1}-p_{i}\right)-\left(p_{i+2}-p_{i-1}\right)\right] .
$$

The discretized continuity equation is expressed as follows

$$
\begin{aligned}
& D_{h}(U)=\frac{\delta_{x} u_{i}}{\Delta x}+\frac{\delta_{y} v_{j}}{\Delta y}, \\
& \delta_{x} u_{i}=\left[27\left(u_{i+1 / 2}-u_{i-1 / 2}\right)-\left(u_{i+3 / 2}-u_{i-3 / 2}\right)\right] /(24 \Delta x), \\
& \delta_{x} v_{j}=\left[27\left(v_{j+1 / 2}-v_{j-1 / 2}\right)-\left(v_{j+3 / 2}-v_{j-3 / 2}\right)\right] /(24 \Delta y) .
\end{aligned}
$$

A brief derivation of Equations (3.1)-(3.7) is given in Appendix A. The method for obtaining pressure will be presented in the next section. In the discretization of pressure gradient and continuity equation, an approximation with reduced formal accuracy is used. This is for the easy solving of the Poisson equation for the pressure. It will be shown in Section 5 that the resolving efficiency of the fourth-order approximation on the cell-centered mesh is almost the same as that for the sixth-order approximation over a wide range of wavenumbers.

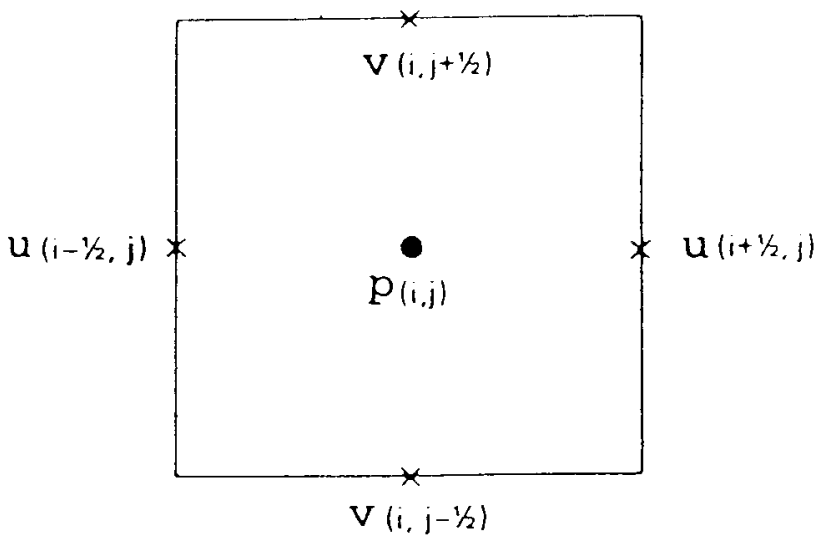

Figure 1. Staggered grid system. 


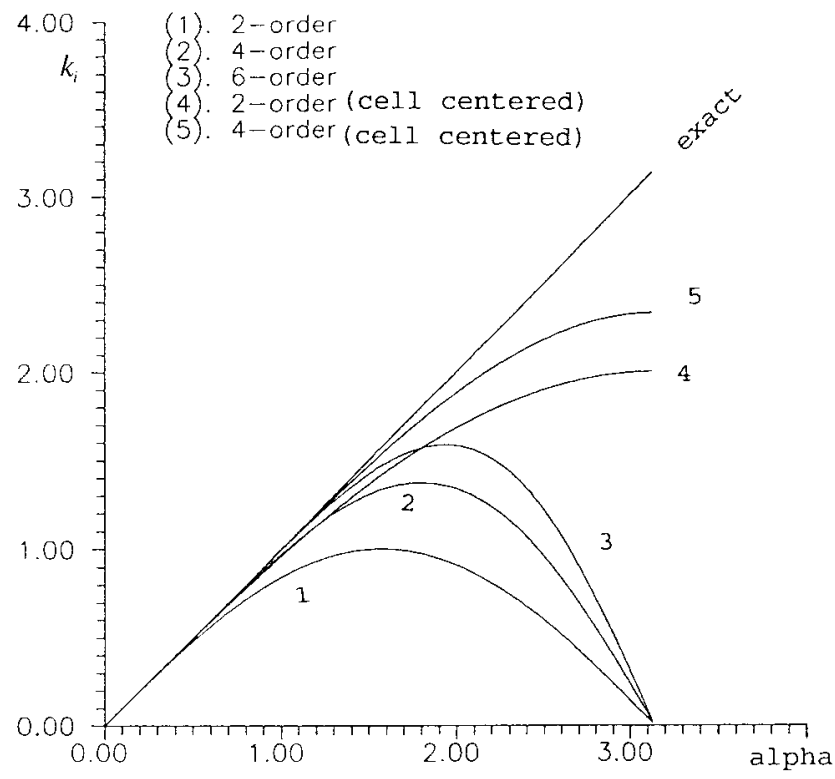

Figure 2. Variation of the modified wavenumbers $k_{i}$ vs. alpha.

In the difference equation for the first momentum equation, the value of the velocity component $v$, which is defined at point $\left(i, j+\frac{1}{2}\right)$, needs to be computed at the point $\left(i+\frac{1}{2}, j\right)$. In the present paper, $v_{i+1 / 2, j}$ is obtained from a sixth-order-accurate midpoint interpolation developed in [5]. In the same way, $u_{i, j+1 / 2}$ can be computed for the second momentum equation.

Suppose all derivatives in (2.8) and (2.9) are discretized. After that, the following semi-discretized approximation for (2.9) is obtained

$$
\frac{\mathrm{d} U}{\mathrm{~d} t}=L_{h}(U)
$$

A three-stage Runge-Kutta $(\mathrm{R}-\mathrm{K})$ method with third-order accuracy [9] is used to discretize the resulting ordinary differential equations

$$
\begin{aligned}
& U^{(1)}=\alpha_{1} U^{n}+\beta_{1} \Delta t L_{h}\left(U^{n}\right), \\
& U^{(2)}=\alpha_{2} U^{n}+\beta_{2}\left[U^{(1)}+\Delta t L_{h}\left(U^{(1)}\right)\right], \\
& U^{n+1}=\alpha_{3} U^{n}+\beta_{3}\left[U^{(2)}+\Delta t L_{h}\left(U^{(2)}\right)\right],
\end{aligned}
$$

where $\alpha_{1}=1, \alpha_{2}=3 / 4, \alpha_{3}=1 / 3, \beta_{1}=1, \beta_{2}=1 / 4$ and $\beta_{3}=2 / 3$.

Table I. Comparison of resolving efficiency

\begin{tabular}{lccccc}
\hline$\varepsilon$ & & & & & \\
$e_{1}$ & 0.100 & 0.050 & 0.010 & 0.005 & 0.001 \\
Scheme & & & & & \\
\hline Cell-centered & 0.74 & 0.61 & 0.39 & 0.33 & 0.22 \\
Fourth-order & 0.44 & 0.37 & 0.24 & 0.20 & 0.13 \\
Sixth-order & 0.54 & 0.47 & 0.35 & 0.31 & 0.23 \\
\hline
\end{tabular}




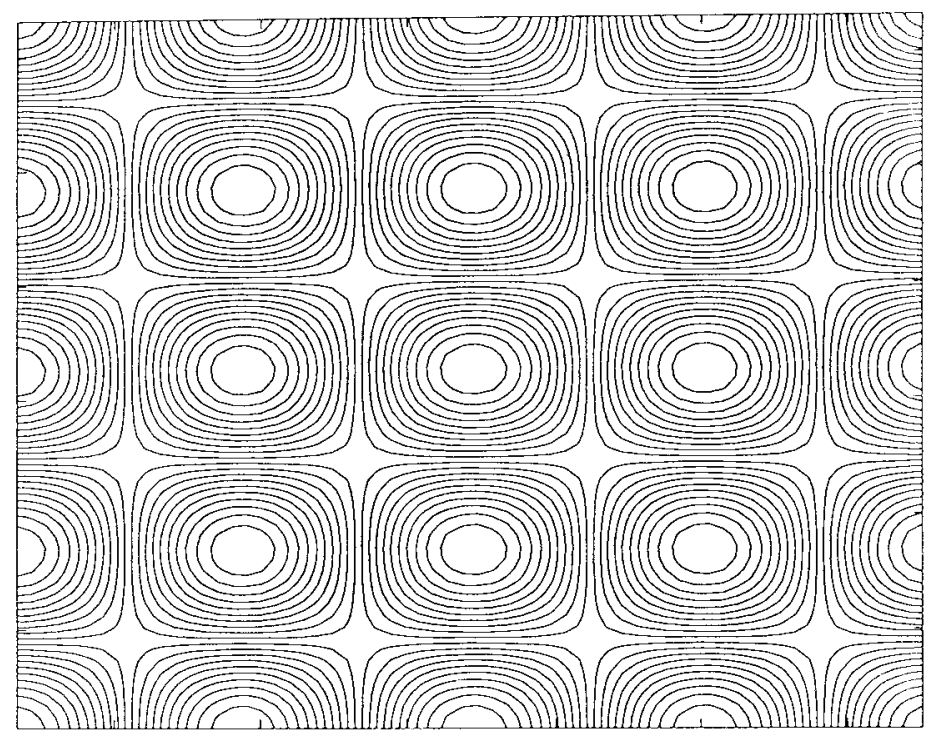

Figure 3. Vorticity contours for the Taylor's vortex at $t=2$.

\section{SOLUTION OF PRESSURE EQUATION}

At each step of the $\mathrm{R}-\mathrm{K}$ method, a Poisson equation for pressure is solved. As a sample solution algorithm, consider the first step of the $\mathrm{R}-\mathrm{K}$ method. Applying the difference operator $D_{h}$ to Equation (3.9) with discretized $f, g, \partial p / \partial x$ and $\partial p / \partial y$ in (2.5)-(2.7), you obtain

$$
D_{h}\left[U^{(1)}\right]=\alpha_{1} D_{h}\left[U^{(n)}\right]+\beta_{1} D_{h}[F]-\beta_{1} D_{h}\left[G_{h}(p)\right],
$$

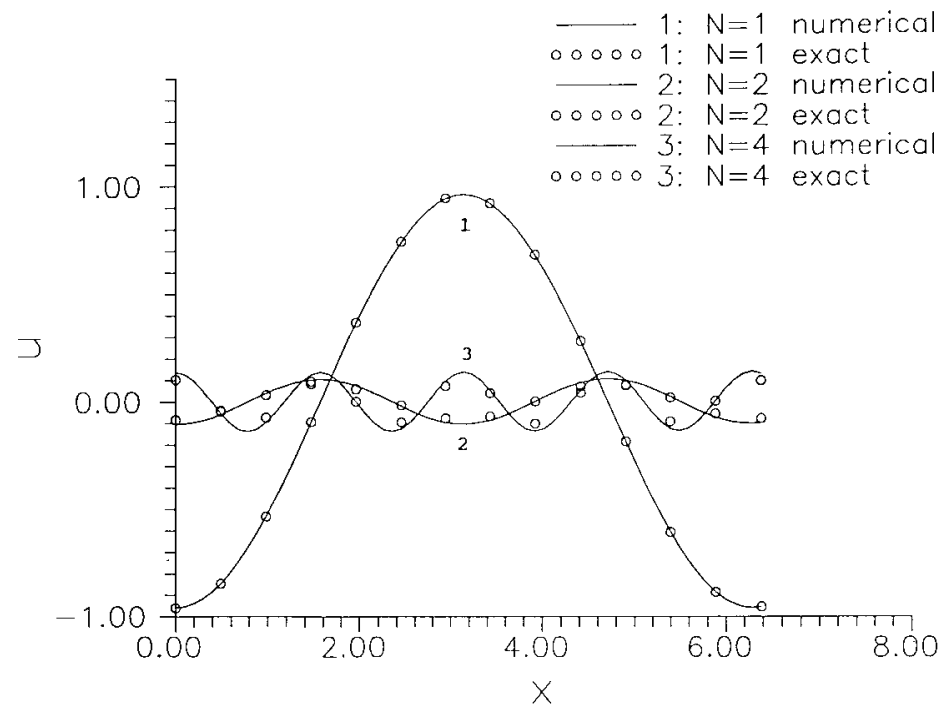

Figure 4. Velocity component $u$ at $J=16$ for $N=1,2,4$ and $R \mathrm{e}=100$. 


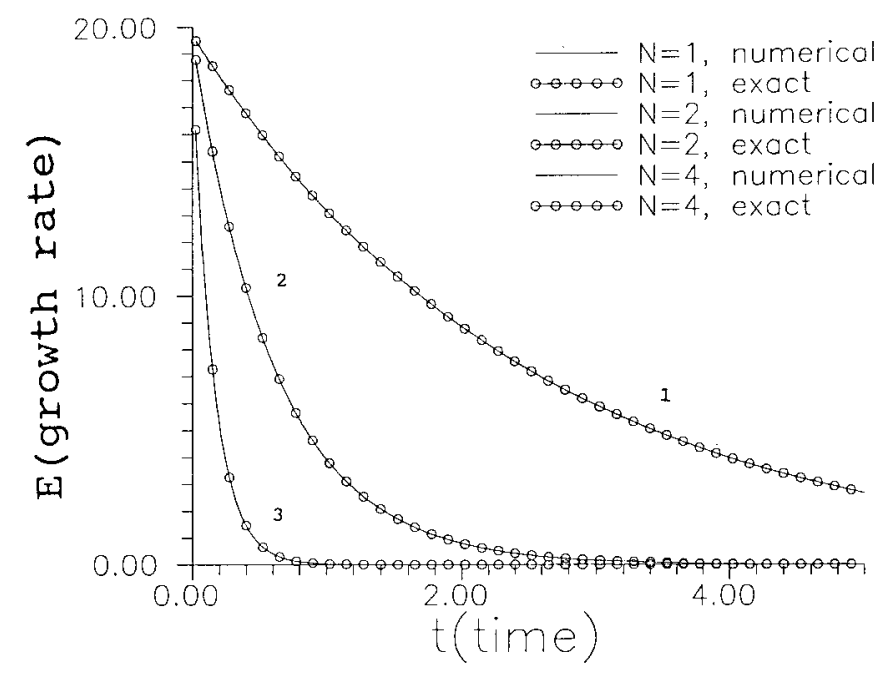

Figure 5. Decay of kinetic energy vs. time for $N=1,2,4$ and $R \mathrm{e}=10$.

where $G_{h}$ is the difference approximation of the pressure gradient. As is done for the commonly used second-order projection method for the incompressible Navier-Stokes equations, suppose $D_{h}\left[U^{(1)}\right]=0$. With this assumption, the following discretized Poisson equation for pressure is obtained from (4.1)

$$
\beta_{1}\left[\frac{\delta_{x}\left(\delta_{x} p\right)}{\Delta x^{2}}+\frac{\delta_{y}\left(\delta_{y} p\right)}{\Delta y^{2}}\right]=\alpha_{1} D_{h}\left(U^{(1)}\right)+\beta_{1} D_{h}[F],
$$

where

$$
\begin{aligned}
& \delta_{x}\left(\delta_{x} p\right)=\frac{1}{(24)^{2}}\left[\left(p_{i+3}+p_{i-3}\right)-54\left(p_{i+2}+p_{i-2}\right)+783\left(p_{i+1}+p_{i-1}\right)-1460 p_{i}\right], \\
& \delta_{y}\left(\delta_{y} p\right)=\frac{1}{(24)^{2}}\left[\left(p_{j+3}+p_{j-3}\right)-54\left(p_{j+2}+p_{j-2}\right)+783\left(p_{j+1}+p_{j-1}\right)-1460 p_{j}\right] .
\end{aligned}
$$

The right-hand-side of Equation (4.2) is known. With periodic boundary conditions in both the $x$ - and $y$-directions, one has a linear system of equations for the pressure with a cyclic block hepta-diagonal matrix. The corresponding matrix is denoted $A$. The obtained linear system of equations with unknown vector $P$ for pressure can be written as

$$
A P=H,
$$

where $H$ is a known vector. The preconditioned system is

$$
A M^{-1} Z=H
$$

where

$$
Z=M P .
$$

First the system (4.6) needs to be solved, and then with $Z$ obtained from (4.6), the solution $P$ is obtained from (4.7). In the present paper, a SSOR preconditioner [10] is used. The precondition matrix $M$ is defined as 


$$
M=(D-\omega E) D^{-1}(D-\omega G),
$$

where $\omega$ is a relaxation parameter, $D$ is a diagonal matrix consisting of the diagonal elements of matrix $A,-E$ is the strict lower part of $A$, and $-G$ is the strict upper part of $A$.

The solution process is now briefly outlined. Suppose $u^{n}, v^{n}$ an $p^{n}$ are known. The process of the solution is as follows

1. Step 1 of Runge-Kutta method

(a) with fifth-order upwind compact difference operators, discretize the convection terms of the Navier-Stokes equations, and with sixth-order symmetric compact difference operator, discretize the viscous terms of equations;

(b) with difference operator $D_{h}(U)$ in (3.5), calculate the right-hand-side of (4.2);
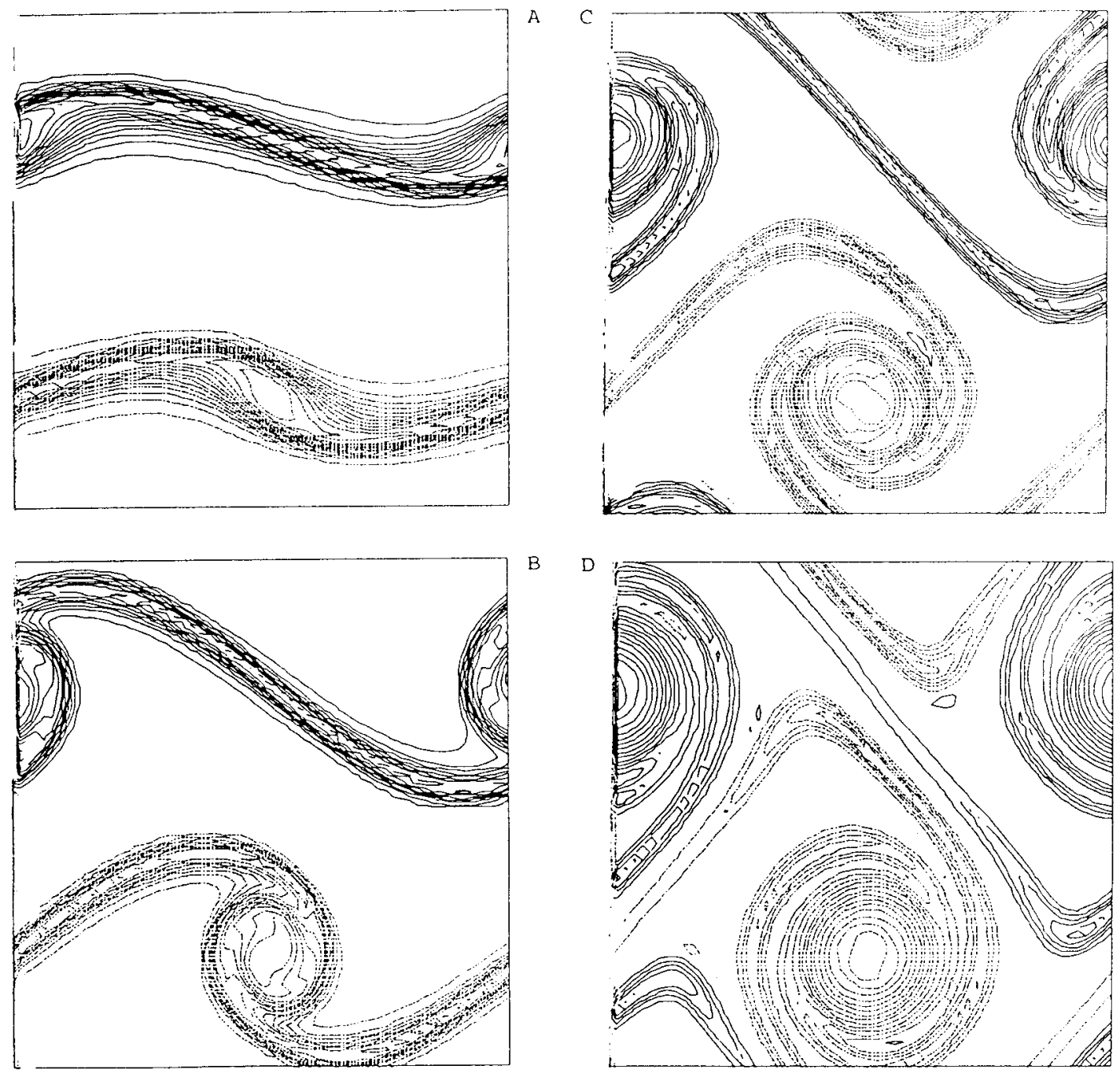

Figure 6. Vorticity contours for $64 \times 64$. A: $t=4$, B: $t=6$, C: $t=8$, D: $t=10$. 

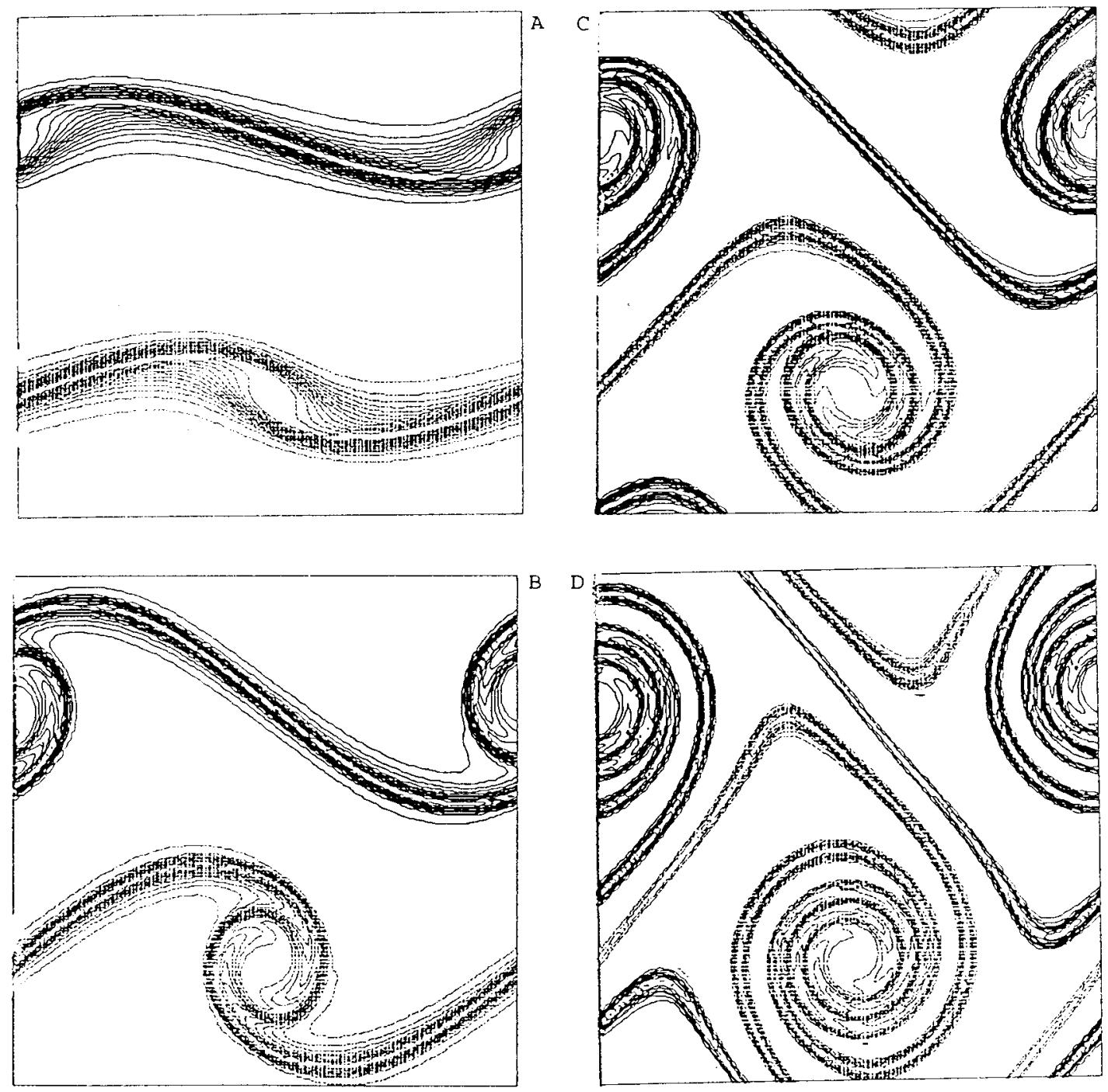

Figure 7. Vorticity contours for $128 \times 128$. A: $t=4$, B: $t=6$, C: $t=8$, D: $t=10$.

(c) solve the Poisson equation (4.2) for obtaining the pressure vector $p^{(1)}$ with the preconditioned method (4.6) and (4.7);

(d) with obtained $p^{(1)}$ and discretization (4.3) and (4.4), compute $U^{(1)}$ in (3.9);

2. Step 2 of Runge-Kutta method: Repeat (a)-(c) in Step 1 with $U^{(1)}$ obtained in Step 1 instead of $U^{(n)}$ to compute $f\left(U^{(1)}\right), g\left(U^{(1)}\right)$ and $P^{(2)}$, and then compute $U^{(2)}$ from (3.10);

3. Step 3 of Runge-Kutta method: Repeat (a)-(c) in Step 1 with $U^{(2)}$ obtained in Step 2 instead of $U^{(1)}$ to compute $f\left(U^{(2)}\right), g\left(U^{(2)}\right)$ and $p^{n+1}$, and then compute $U^{n+1}$ from (3.11).

4. Repeat process $1-3$. 


\section{ACCURACY ANALYSIS}

It was shown is [5,6] that compact schemes and upwind compact schemes have higher resolving efficiency than traditional schemes. Compared with traditional fifth-order upwind-biased scheme, the fifth-order upwind compact scheme has less dissipation over a wider range of wavenumbers and larger dissipation at very high wavenumbers [6]. Less dissipation provides dominance of physical dissipation over a wider range of wavenumbers. Larger numerical dissipation at very high wavenumbers is useful for suppressing potential numerical oscillations. Reduced formal accuracy was used in the approximation of the pressure gradient and the continuity equation. In this section, it is shown that the adopted fourth-order difference approximation on a cell-centered mesh has almost the same resolving efficiency over a wide range of wavenumbers as the sixth-order traditional difference approximation. Consider the following three difference approximations:

Traditional fourth-order approximation

$$
F_{j}=\frac{1}{12}\left[8\left(u_{j+1}-u_{j-1}\right)-\left(u_{j+2}-u_{j-2}\right)\right] .
$$

Traditional sixth-order approximation

$$
F_{j}=\frac{1}{60}\left[45\left(u_{j+1}-u_{j-1}\right)-9\left(u_{j+2}-u_{j-2}\right)+\left(u_{j+3}-u_{j-3}\right)\right] .
$$

Fourth-order approximation on a cell-centered mesh

$$
F_{j}=\frac{1}{24}\left[27\left(u_{j+1 / 2}-u_{j-1 / 2}\right)-\left(u_{j+3 / 2}-u_{j-3 / 2}\right)\right] .
$$

The corresponding modified wavenumbers are given in Figure 2. The range of well-resolved wavenumbers may be defined by the error tolerance [5]

$$
\left|\frac{k_{i}(\alpha)-\alpha}{\alpha}\right|<\varepsilon,
$$

where $k_{i}(\alpha)$ is the modified wavenumber. Suppose $\alpha_{f}$ is the shortest well-resolved wavenumber. The fraction $e_{1}=\alpha_{f} / \pi$ may be regarded as a measure of the resolving efficiency of a scheme. From Figure 2, it can be seen that the fourth-order approximation on the cell-centered mesh can also give good resolving efficiency. With different error tolerance, the comparison of the resolving efficiency for approximation (5.1)-(5.3) is given in Table I. From the table, it can be seen that the fourth-order difference approximation on the cell-centered mesh has almost the same resolving efficiency as the sixth-order approximation over a wide range of wavenumbers.

\section{NUMERICAL EXAMPLES}

Example 1. Taylor in 1923 published a exact solution of the incompressible Navier-Stokes equations in terms of the streamfunction and vorticity [11]. In the present paper, the initial condition is taken as [12]

$$
\begin{aligned}
& u(x, y, 0)=-\cos (N x) \sin (N y), \\
& v(x, y, 0)=\sin (N x) \cos (N y), \\
& 0 \leq x \leq 2 \pi, \quad 0 \leq y \leq 2 \pi .
\end{aligned}
$$


The exact solution for the this case is known:

$$
\begin{aligned}
& u(x, y, t)=-\cos (N x) \sin (N y) \exp \left[-2 N^{2} t / R e\right], \\
& v(x, y, t)=\sin (N x) \cos (N y) \exp \left[-2 N^{2} t / R e\right],
\end{aligned}
$$

where $N$ is a integer. The computed Taylor's array of vortices for $\Delta x=\Delta y=2 \pi / 64, R \mathrm{e}=100$ and $N=2$ at $t=2$ is given in Figure 3. The variation of velocity component $u$ with $x$ at $y=\pi / 2$ for $N=1,2,4$ is given in Figure 4. The difference between the exact solution and predicted solution from the simulation for the case using a coarse mesh grid, $\Delta x=\Delta y=2 \pi / 16, R \mathrm{e}=100$ at $t=2$, is within a maximum difference of $10^{-3}$. Comparison of the computationally predicted decay of kinetic energy with that of the exact solution is given in Figure 5 for the case $R \mathrm{e}=10, N=1,2,4$.

Example 2. This is a double layer taken from [13].

$$
\begin{aligned}
& u(x, y, 0)= \begin{cases}\tanh [(y-\pi / 2) / \rho], & y \leq \pi \\
\tanh [(3 \pi / 2-y) / \rho], & y>\pi\end{cases} \\
& v(x, y, 0)=\delta \sin (x),
\end{aligned}
$$

where $\rho=\pi / 15, \delta=0.05, R \mathrm{e}=10^{4}$. The incompressible Navier-Stokes equations with periodic boundary conditions are used. The grid system used in the computation includes $I N \times J N=64 \times 64$ and $128 \times 128$. The results at different times are given in Figures 6 and 7 . The purpose of this computation is to test the efficiency of method to solve a problem with a steep gradient in the solution.

\section{ACKNOWLEDGMENTS}

This work is supported by State Key Project, NNSFC, JSPS and Network Information Centre, CAS.

\section{APPENDIX A}

This appendix presents a brief derivation of the upwind compact difference approximation. Consider the following model equation and its semi-discrete approximation

$$
\begin{aligned}
& \frac{\partial u}{\partial t}+c \frac{\partial u}{\partial x}=0, \quad c=\text { constant } \\
& \frac{\partial u_{j}}{\partial t}+c \frac{F_{j}}{\Delta x}=0
\end{aligned}
$$

where $F_{j} / \Delta x$ is an approximation of the first derivative $\partial u / \partial x$, and $F_{j}$ is obtained from the following difference equation

$$
\alpha_{0} F_{j+1}+\beta_{0} F_{j}+\gamma_{0} F_{j-1}=a\left(u_{j+2}-u_{j+1}\right)+b\left(u_{j+1}-u_{j}\right)+c\left(u_{j}-u_{j-1}\right)+d\left(u_{j-1}-u_{j-2}\right),
$$

with the requirement

$$
\alpha_{0}+\beta_{0}+\gamma_{0}=1
$$


The relations between the coefficients $\alpha_{0}, \beta_{0}, \gamma_{0}, a, b, c$ and $d$ are derived by matching the Taylor series coefficients of various orders. The first unmatched coefficient divided by $\Delta x$ determines the formal truncation error. The constraints are

$$
\begin{aligned}
& \alpha_{0}+\beta_{0}+\gamma_{0}=1, \quad a+b+c+d=1, \\
& \alpha_{0}-\gamma_{0}=(3 a+b-c-3 d) / 2, \alpha_{0}+\gamma_{0}=(7 a+b+c+7 d) / 3, \\
& \alpha_{0}-\gamma_{0}=(15 a+b-c-15 d) / 4, \alpha_{0}+\gamma_{0}=(31 a+b+c+31 d) / 5 .
\end{aligned}
$$

There are seven unknowns with six equations. The following relations with one free parameter can be obtained from (A4)

$$
\begin{aligned}
& \beta_{0}=3 / 5, \quad a=\alpha_{0} / 6-1 / 60, \quad c=-3 / 2 \alpha_{0}+47 / 60, \quad \gamma_{0}=2 / 5-\alpha_{0}, \\
& b=3 \alpha_{0} / 2+11 / 60, \quad d=-\alpha_{0} / 6+1 / 20 .
\end{aligned}
$$

With the initial condition

$$
u(x, 0)=\exp (i k x)
$$

(A1) has an exact solution

$$
u(x, 0)=\exp [i k(x-c t)] .
$$

With the same initial condition (A6), one can obtain the solution of (A2)

$$
u\left(x_{j}, t\right)=\exp \left[-\frac{k_{r}}{\Delta x} c t\right] \exp \left[i k\left(x_{j}-c \frac{k_{i}}{\alpha} t\right)\right],
$$

where $0 \leq \alpha=k \Delta x \leq \pi$,

$$
\begin{aligned}
& k_{i}(\alpha)=\frac{B C-A D}{C^{2}+D^{2}}, \quad k_{r}(\alpha)=\frac{A C+B D}{C^{2}+D^{2}}, \\
& A=5\left(\alpha_{0}-0.2\right)[\cos (2 \alpha)+8 \cos (\alpha)-9], \\
& B=[\sin (2 \alpha)+28 \sin (\alpha)] / 6, \\
& C=3+2 \cos (\alpha), \\
& D=10\left(\alpha_{0}-0.2\right) \sin (\alpha) .
\end{aligned}
$$

With $\alpha_{0}=0.2$, we have $k_{r}(\alpha)=0$, and (A3) is the sixth-order-accurate symmetrical compact difference approximation. Suppose $c>0$ in (A1). $k_{r}(\alpha)$ with $\alpha_{0}<0.2$ is positive, and the scheme (A2) is dissipative (see (A8)). The following simplest fifth-order dissipative (or upwind) compact approximation can be obtained if $\alpha_{0}=0$ is taken

$$
\frac{3}{5} F_{j}+\frac{2}{5} F_{j-1}=\frac{1}{60} \delta_{x}^{-}\left[-u_{j+2}+11 u_{j+1}+47 u_{j}+3 u_{j-1}\right] .
$$

Equation (A10) can be solved easily with increasing the index $j$. The difference relations (3.2)-(3.4), (3.6) and (3.7) can be obtained in the same way. 


\section{REFERENCES}

1. R. Rogallo and P. Moin, 'Numerical simulation of turbulent flows', Annu. Rev. Fluid Mech., 16, 99-137 (1984).

2. J. Kim, P. Moin and R.D. Moser, 'Turbulence statistics in full developed channel flow at low Reynolds number', J. Fluid Mech., 177, 133-166 (1987).

3. P.R. Spalart, 'Direct simulation of a turbulent boundary layer up to $R \mathrm{e}=1410$ ', J. Fluid Mech., 187, 61-98 (1988).

4. M.M. Rai and P. Moin, 'Direct simulation of turbulent flow using finite difference schemes', J. Compt. Phys., 96, 15-53 (1991).

5. S.K. Lele, 'Compact finite difference schemes with spectral-like resolution', J. Comput. Phys., 103, 16-42 (1992).

6. Fu Dexun and Ma Yanwen, 'High resolution scheme', in M. Hafez and K. Oshima (eds.), Computational Fluid Dynamics Review 1995, Wiley, 1995, pp. 234-250.

7. Ma Yanwen and Fu Dexun, 'Super compact finite difference method (SCFDM) with arbitrarily high accuracy', Comput. Fluid Dyn. J., 5, 259-276 (1996).

8. Fu Dexun and Ma Yanwen, 'A high-order-accurate difference scheme for complex flow fields', J. Comput. Phys., 134, 1-15 (1997).

9. C.-W. Shu and S. Osher, 'Efficient implementation of essentially non-oscillatory shock-capturing schemes', $J$. Comput. Phys., 77, 439-471 (1988).

10. Yousef Saad, 'Preconditioned Krylov subspace methods: an overview', in M. Hafez and K. Oshima (eds.), Computational Fluid Dynamics Review 1995, Wiley, 1995, pp. 437-456.

11. G.I. Taylor, 'On the decay of vortices in a viscous fluid', Philos. Mag., 46, 671-674 (1923).

12. A. Chorin, 'Numerical solution of the Navier-Stokes equations', Math. Comput., 22, 745-762 (1968).

13. J. Bell, P. Colella and H. Gloz, 'A second-order production method for the incompressible Navier-Stokes equations', J. Comput. Phys., 85, 257-283 (1989). 